\title{
Decision-Making Heuristics for Managing Climate-Related Risks: Introducing Equity to the FREE Framework
}

\author{
Camilla Audia, Emma Visman, Gino Fox, \\ Emmah Mwangi, Mary Kilavi, Mark Arango, Sonja Ayeb- \\ Karlsson, and Dominic Kniveton
}

\begin{abstract}
Managing climate-related risks is clouded in differing levels of uncertainty that are magnified when trying to understand their potential impacts on socio-ecological systems. The 'cascade of uncertainty' is
\end{abstract}

C. Audia $(\bowtie)$

Department of Geography, King's College London, London, UK

e-mail: camilla.audia@kcl.ac.uk

E. Visman

UK Centre for Ecology and Hydrology, Wallingford, UK

King's College London, London, UK

G. Fox $\bullet$ S. Ayeb-Karlsson • D. Kniveton

University of Sussex, Brighton, UK

E. Mwangi $\bullet$ M. Arango

Kenya Red Cross Society, Nairobi, Kenya

M. Kilavi

Kenya Meteorological Department, Nairobi, Kenya

(C) The Author(s) 2021

D. Conway, K. Vincent (eds.), Climate Risk in Africa, https://doi.org/10.1007/978-3-030-61160-6_4 
particularly apparent in Africa where socio-ecological data are sparse, and the development and validation of impact models are at varying stages. In this context, using heuristics may serve as an effective way for policy makers to incorporate climate change knowledge into decision-making. Previous scholarship has identified the principles of Flexibility, Robustness and Economic low/no regrets in decision-making under uncertainty. In this chapter, we first make the case for adding Equity to these heuristics, where equity involves ensuring that reducing the climate change risk for one cohort of society does not result in its increase for another. Second, we describe how these principles have been applied under two DFID/ NERC funded projects: ForPAc and AMMA-2050 through the use of Participatory Impact Pathways Analysis tools.

Keywords Climate change $\bullet$ Heuristics $\bullet$ Uncertainty $\bullet$ Equity $\bullet$ Decision-making

\section{INTRODUCTION}

Attempts to reduce climate risks on society need to consider the issue of uncertainty. Weather and climate ${ }^{1}$ information has the potential to inform climate-risk management efforts drawing from historical observations, model ensembles of current climate, through short-term weather forecasts to seasonal forecasts and future climate scenarios. Different climate information has different degrees and sources of uncertainty. For example, short-term (e.g. daily to weekly) forecasts are inherently probabilistic due to the atmosphere's chaotic nature, while uncertainty at climate change timescales arises from model uncertainty, emission uncertainty and natural climate variability (Stainforth et al. 2007; Chap. 2). This uncertainty not only varies with the lead-time of the forecast or projection but the parameter of interest, region and the spatial and temporal scale of forecasting product.

The different uncertainty levels in forecasts and scenarios of future weather and climate magnify when trying to understand the impact these changes will have on socio-ecological systems (Daron et al. 2015). The

\footnotetext{
${ }^{1}$ Hereinafter weather and climate will be referred to simply as 'climate'.
} 
compounded effects of uncertainty on efforts to identify risk and adaptation options are often referred to as the 'cascade of uncertainty' (Wilby and Dessai 2010). The cascade of uncertainty is particularly apparent in West and East Africa where socio-ecological data are relatively sparse and development and validation of impact models are at varying stages. This uncertainty often results in the view that resolving climate-related risk is a complex and even wicked problem where the solutions are not easily, if at all, solved analytically. In the context of such complex problems, decisionmaking using heuristics (approximate guidelines based on experience) use climate science to bound rather than optimise decisions. Using heuristics to inform decisions often serves as the most effective and sometimes only way for policy and decision-makers to incorporate climate information and knowledge into their thinking and action.

Previous scholarship has identified the principles of Flexibility, Robustness and Economic low-regrets when making decisions within the context of uncertainty (Wilby and Dessai 2010; Ranger et al. 2013; Maier et al. 2016). Accordingly, the principle of Flexibility involves making decisions that can be changed as new climate information evolves; Robustness involves decisions that may lead to positive outcomes across a range of scenarios and forecasts; and Economic low regrets decisions are ones that attempt to negate the possibility of minimal or zero returns in the future at the expense of investment in other priorities in the present (Ranger et al. 2013).

Here we firstly make the case for adding Equity to these principles to make the mnemonic FREE (Flexible, Robust, Economic no/low Regrets, Equitable) to guide decision-making around climate risk. We focus on equity both in ensuring that reducing the weather and climate change risk for one cohort of society or one element of the ecosystem does not result in transferring or increasing risks to another and in its role and value in inclusive decision-making. The second part of the chapter describes Participatory Impact Pathways Analysis (PIPA) and considers the extent to which this approach has been able to support application of the FREE principles, leading to more equitable and inclusive decision-making across timeframes, within two research projects: Towards Forecast-Based Preparedness Action (ForPAc, https://www.forpac.org/) and African Monsoon Multidisciplinary Analysis-2050 (AMMA-2050, https://www. amma2050.org/). 


\section{The FreE Framework of Heuristic Decision-Making}

The Intergovernmental Panel on Climate Change (IPCC) states in its Fifth Assessment Report (WGII AR5) that in Africa 'Climate change [will be] a multiplier of existing health vulnerabilities (high confidence), including insufficient access to safe water and improved sanitation, food insecurity, and limited access to health care and education' (Niang et al. 2014, p. 1202). The IPCC places Climate Risk Management at the centre of attempts to adapt to climate change impacts such as these. Integrated in the concept of 'risk' is the acknowledgement that future climate change is uncertain. Uncertainty in planning for climate change arises not only from our incomplete knowledge of climate processes and the inability to model them but also from unknowns as to which pathways society will choose in terms of the emissions of greenhouse gases. Uncertainty also arises in how climate-related stresses and shocks will impact socio-ecological systems and how these will respond to, reduce, or magnify risk.

A variety of strategies are being used in African countries to manage the impacts of climate-related hazards at the household, community, national and regional levels. These include early warning systems, risk transfer schemes, social safety nets, disaster risk contingency funds and budgeting, livelihood diversification and migration (Niang et al. 2014; UNISDR 2011). Climate science offers both short-term forecasts and mid- and long-term scenarios of climate hazards to help inform these strategies. However, even in cases where the climate science is relatively advanced and the frequency and intensity of climate hazards are quantifiable (e.g. short-lead weather forecasts), climate science is unable to eliminate all the uncertainty. For example, there is still uncertainty associated with quantifying the climate hazard impact on socio-ecological systems. Furthermore, it is recognised that many of the impacts of climate variability and change are indirect, interconnected and poorly quantified. In this context, a set of common assumptions may help, and heuristic reasoning can be employed (Preston et al. 2015).

Heuristics are commonly used in decision-making when the problem is complex and does not lend itself to linear analytical approaches that attempt to calculate the optimal and most economically efficient solution to a problem. Instead, heuristic-based decision-making aims to support practical operationalisation and effectiveness. Commonly, decision-making heuristics, or rules of thumb, are developed individually, based on actors' framings, experience and knowledge (including scientific knowledge), but 
are also discussed collectively and can evolve through social learning (Agrawal et al. 2009). While rules of thumb are usually based on experience and strongly influenced by scientific evidence, they are also formalised and critiqued in grey literature such as practitioners' guides, policy documents and in peer-review articles (Lorenzoni et al. 2000; Preston et al. 2015 ). The following section outlines four principles for framing heuristicbased decision-making to reduce climate-related risks.

\section{Flexible, Robust, Economic No/Low Regrets and Equitable (FREE)}

A key aspect of heuristic-based decision-making for climate risks is the ability to link short-term actions to longer-term pathways, which is at the core of sustainable climate-resilient planning. Policy makers and governments tend to have more political will to act when faced with a disaster, and that is no different in climate-related emergencies. However, governments are often faced with longer-term planning decisions, including infrastructure and spatial planning. In such contexts, flexibility is strengthened through engaging with diverse stakeholders across scales and managing time-sensitive decisions in ways that, at the same time, support sustainable adaptation plans. Yet this is constrained by uncertainty regarding the scale and direction of future climatic changes and variabilities (Pielke et al. 2012). This uncertainty leads to multiple and diverse possible consequences on complex socio-ecological systems (Daron et al. 2015). Ranger et al. (2013) suggest that adaptation pathways should be able to cope with climate risks in uncertain future scenarios by building flexibility to change over time as more is learned or conditions change.

Recognising the deep uncertainty entailed in managing climate-related risks also underscores the importance of robust reasoning in decisionmaking. The principle of 'Robustness' requires that risk management strategies should perform well against most sets of future conditions and ideally include options for several contexts (Ranger et al. 2013). Decisions may need to account for a range of forecasted conditions that span an important threshold. In West Africa, scenarios of future rainfall change span both an increase and a decrease in rainfall. However, while at first sight this might seem to present too wide a range of possible futures for planning, research by AMMA-2050 has shown that due to the unidirectional increase in temperatures with climate change, irrespective of the sign of rainfall change, crop yields will likely decrease with climate change 
(Roudier et al. 2011; Sultan et al. 2019). Despite this seeming certainty, the magnitude of future change is still highly uncertain.

Heuristics for managing climate-related risks also need to consider the degree to which proposed action supports no or low regrets (collectively termed 'Economic low regrets'), addressing both current and future risks or providing co-benefits for other issues of concern. The IPCC defines no-regrets options as plans or policies that can generate socio-economic benefits whether forecasted climate changes occur or not. For the purpose of this chapter, economic low and no regrets options focus on actions that acknowledge current economic limitations while offering opportunities to build future resilience (Watkiss et al. 2015). For example, natural ecosystem-based flood control exemplifies a low or no regrets options where there are immediate environmental benefits irrespective of future climate change. However, other actions may entail significant trade-offs between objectives and sectors, such as conflicting demands between environmental protection and the need for new housing or economic development. Ranger et al. (2013) suggest that a holistic approach, whereby adaptation planning is mainstreamed into decision-making across different levels and sectors of the government, is a way forward. It is also important that adaptation is not seen in isolation but as part of sustainable development, where potential synergies and trade-offs are considered across a broad range of risks, opportunities, objectives, measures, and timeframes. Consideration of low and no regrets actions across timeframes enables short-term actions to be considered as part of a longer-term preparedness and sustainable adaptation planning processes.

Robust, flexible, and economically low and no regrets decision-making heuristics provide the basis for a comprehensive approach to address the complexity of climate change adaptation planning. These approaches have largely been framed within the context of economic capacity. However, it is unlikely that economic investments or interventions will effectively address climate risk management unless they can characterise how direct and indirect risks, costs and benefits are distributed within a society and across an ecosystem. It is widely acknowledged that economic analyses of the costs and benefits of potential interventions need to be combined with social analyses to understand the potential and sometimes unexpected impacts of planned activities. In making explicit the trade-offs between sectors, timeframes and social groups, heuristics to support climate risk management must also include the principle of equity. Recognising the 
importance of inclusivity, the equity heuristic ensures that climate risks are not simply passed onto more marginalised members of society, displaced to other components of the social-ecological system, or indeed transferred from current to future generations.

Inequities are exacerbated by climate extremes. The literature on disasters and development recommends that factors such as gender, age, race, and ethnicity as well as socio-economic status and social capital are key to individual and collective vulnerability to disasters (Shreve 2016). Such factors, in turn, influence the ability to benefit from interventions and ultimately the capacity to be resilient in the face of climate change. Successful and sustainable climate risk management will ultimately depend on how different institutions address equity, considering social and cultural contexts, representing all at-risk groups, and recognising the diverse ways in which people may be affected by climate-related risks and adaptation interventions. The next section explores how FREE factors have been used in different projects to support sharing climate information between science and policy actors.

\section{Communicating Climate Information Across Science AND POLICY AND FREE}

It is widely recognised that climate information uptake is limited in many developing countries. The lack of uptake has previously been attributed to the 'usability gap' (Lemos et al. 2012). A variety of reasons for the usability gap have been put forward (see also Chaps. 1-3), including

- a lack of credibility, salience, and legitimacy of climate information and climate information producers (Cash et al. 2003);

- a lack of capacity, institutional arrangements and resources amongst users to capitalise on this information (Lorenz et al. 2017);

- mismatched terminology used by scientists and decision-makers to describe the types of information that are available and needed for problem solving (Daly and Dilling 2019);

- unrealistic expectations regarding the development of climate information products for problem solving (Briley et al. 2015); and

- non-conducive organisational culture and individual reward structures to using climate information (Dilling and Lemos 2011). 
Equally a number of facilitating processes, structures and actors to improve the use of climate information have been described, including co-development, co-production, knowledge networks, social learning, and communities of practice (Chap. 3; Lemos et al. 2012; Leitch et al. 2019); as well as information brokers, boundary organisations and chains, embedded capacity and collaborative group processes (Dilling and Lemos 2011; Kirchhoff et al. 2015). Scholars have discussed barriers and opportunities to close the usability gap and increasing attention has been paid to describing the steps needed to help facilitate the use of climate information and knowledge (Singh et al. 2018; Carter et al. 2019), including addressing inequities in the partnerships and processes employed (Daly and Dilling 2019; Vincent et al. 2018; Turnhout et al. 2020). This section considers the application of FREE heuristics in enabling emerging climate science to strengthen climate-resilient decision-making using PIPA, as illustrated within the two projects, ForPAc and AMMA-2050.

\section{Participatory Impact Pathways Analysis (PIPA)}

Designed to enhance a project's developmental impact through better impact assessment, ${ }^{2}$ PIPA is a project management tool that enables stakeholders affected by research to jointly identify a shared vision of the impact of the research and co-develop pathways to achieving it. As a first step, it aims to understand the determinant causes of a research problem from multiple perspectives with participants from a range of stakeholders developing problem trees of the issue(s) in focus. The incorporation of a diversity of stakeholders is key to attempts to incorporate equity into the research. By including marginalised groups, the issue of how negative outcomes can be transferred from one group to another can be explored, and hence the issue of equity raised. Participants subsequently undertake a visioning exercise, designed to agree on an overarching aim of continued engagement. The stakeholders then develop network maps, firstly depicting existing relationships between multiple stakeholder types, before creating 'future' network maps, identifying additional actors and stakeholder linkages required to achieve the shared project vision. The process makes explicit the project's impact pathways, developing an Outcome Logic Model identifying the changes in practice, knowledge, attitudes and skills

\footnotetext{
${ }^{2}$ Link to the PIPA wiki page: http://pipamethodology.pbworks.com/w/page/ $70283575 /$ Home\%20Page
} 
required to achieve the shared project aim. The PIPA process offers a vital opportunity for joint reflection between researchers and societal partners on how to scale co-production products and processes out across relevant institutions and up, supporting transformative changes through social learning, advocacy and policy change.

PIPA is well-suited to supporting inclusive approaches to strengthening climate resilience. The approach (1) recognises the need to listen to people's different framings of the risks that climate poses; (2) encourages inclusive participation in decision-making; and (3) co-develops pathways to achieve strengthened climate-resilience (Fox and Kniveton 2018). The approach is sufficiently flexible to be adapted with additional and complementary methodologies and can be modified according to the goals at a certain point of a project. This allows for several iterations of PIPA over the course of the project, each creating spaces for formal and informal discussions that lead to collective and transactional decision-making. The following sections explore how PIPA has supported flexible, robust, economic (no and low regrets) and equitable climate-resilient decisionmaking in projects focused on differing timescales in both urban and rural contexts in Kenya and Burkina Faso.

\section{Towards Forecast-Based Preparedness and Action}

Guided by the Sendai Framework for Disaster Risk Reduction, humanitarian organisations are increasingly seeking to enhance mitigation and preparedness for climate-related risks. Forecast-based Action $(\mathrm{FbA})$ is a set of loosely associated approaches to support the use of forecasts in undertaking relevant early actions for at-risk communities in resource-constrained contexts. They are similar in design to early warning systems in terms of forecasting and communication of possible threats but place more emphasis on protocols, so actors know what to do based on a range of forecasts (Wilkinson et al. 2018). Recognising the inherent uncertainty in forecasting potential disasters, $\mathrm{FbA}$ approaches attempt to help decision-makers take into account the costs and benefits of anticipatory actions and forecast-driven false alarms. While $\mathrm{FbA}$ approaches attempt in theory to provide an economically defensible rationale to making a decision on whether to invest in preparedness or mitigation actions based on a forecast, in practice they are often difficult to implement because disasters tend to be unique and the losses, including the quantification of cascading risks, difficult to determine. 
Funded by the Science for Humanitarian Emergencies and Resilience (SHEAR) Research Programme, ForPAc seeks to improve forecasts at different lead times and strengthen forecast-based action for flood and drought hazards in Kenya. It seeks to support anticipatory decisionmaking in three case studies: (i) the Drought Early Warning System (DEWS) in Kitui County, (ii) urban flooding in Nairobi and (iii) the flood early warning system in the Nzoia river basin. In a series of workshops, PIPA was employed to consider how climate forecasts can better support existing drought preparedness decision-making for Kitui County. This follows the national DEWS process but is managed by a County Steering Group (CSG), comprising the National Drought Management Authority (NDMA), key ministries of the Kitui County government, humanitarian and development partners and the Kenya Meteorological Department County Director of Meteorological Services.

Alongside problem tree analysis, visioning and stakeholder mapping to strengthen researchers' understanding of the decision-making context, PIPA and a subsequent climate information training workshop included exercises to strengthen decision-makers' understanding of key climate concepts, including forecast uncertainty, as well as their confidence in using probabilistic forecasts within drought decision-making. Drawing on Participatory Integrated Climate Services for Agriculture (PICSA) (Dayamba et al. 2018) and the principle of Economic no/low regrets, the project also integrated within PIPA a tailored preparedness options matrix. Mapping initial phases of drought and forecast timeframes with preparedness actions and levels of investment to identify the forecast probability thresholds required to activate actions (see Table 4.1) highlighted the potential for triggering low-cost preparedness actions (e.g. awarenessraising, advocacy and prepositioning of stocks) at longer lead times. Discussion on the completed matrices made clear that marginal mixed farming in arid areas experiences water scarcity even in seasons of 'normal' rains, requiring minimal probabilities of below normal forecasted rainfall to justify investment in preparedness.

PIPA employed a tailored version of stakeholder mapping, with participants identifying the key steps in the drought decision-making process, the actors engaged, and climate information being employed at each step in the process. This mapping made clear that the climate information is not currently informing several key steps within the DEWS process, including drought contingency planning, monthly bulletins and seasonal assessments. Operationalising the principles of flexibility and robustness, 


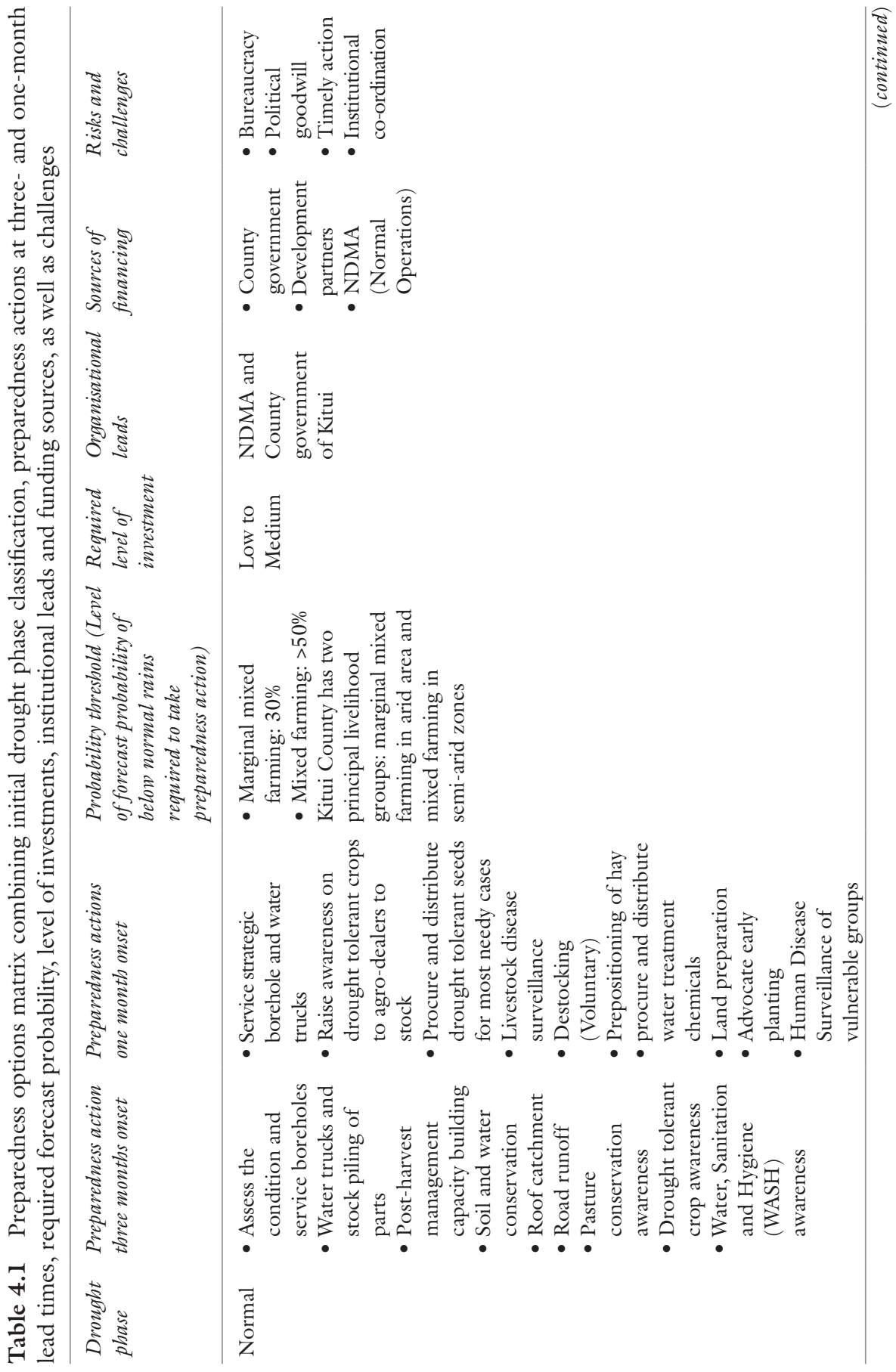




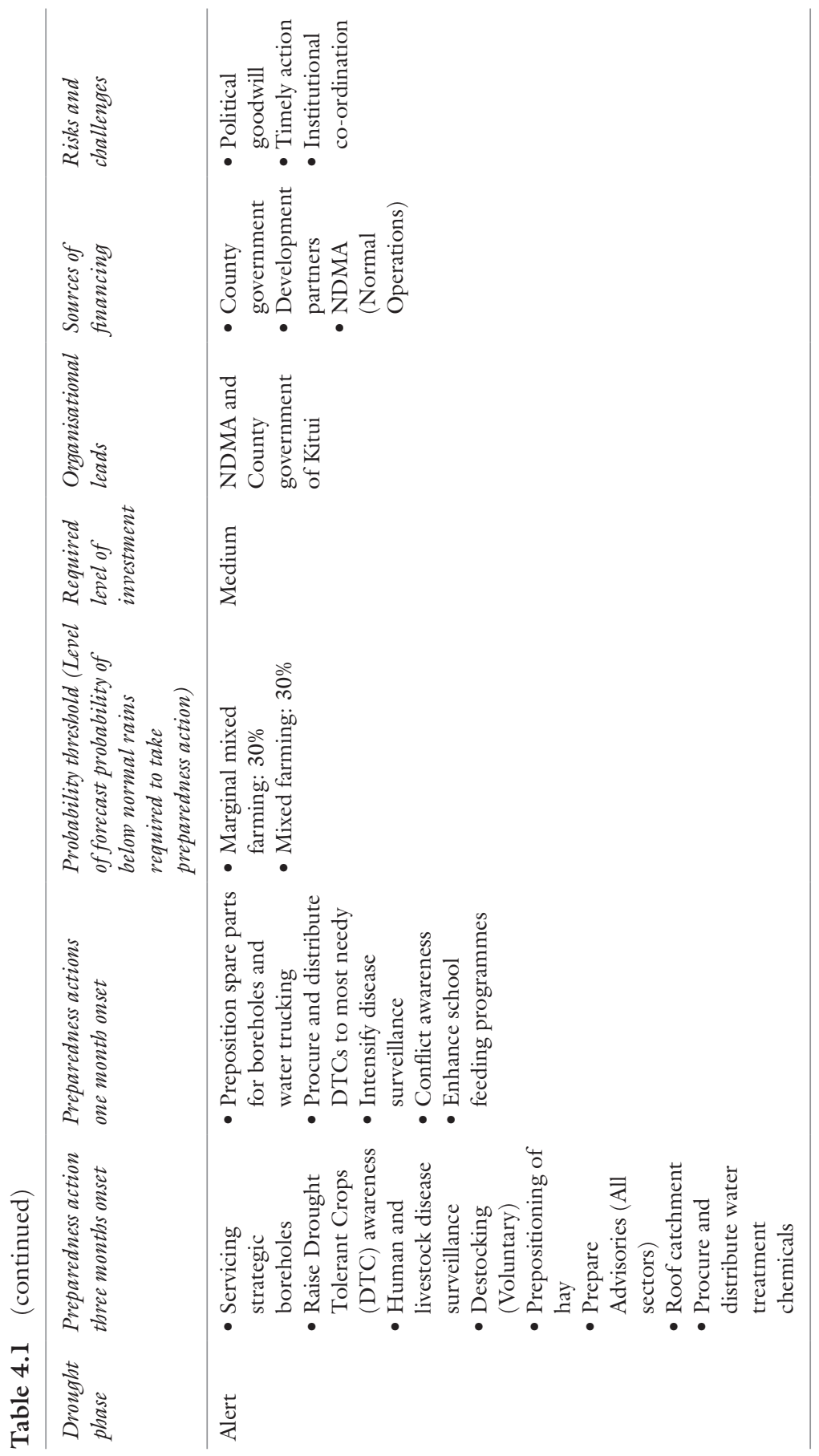




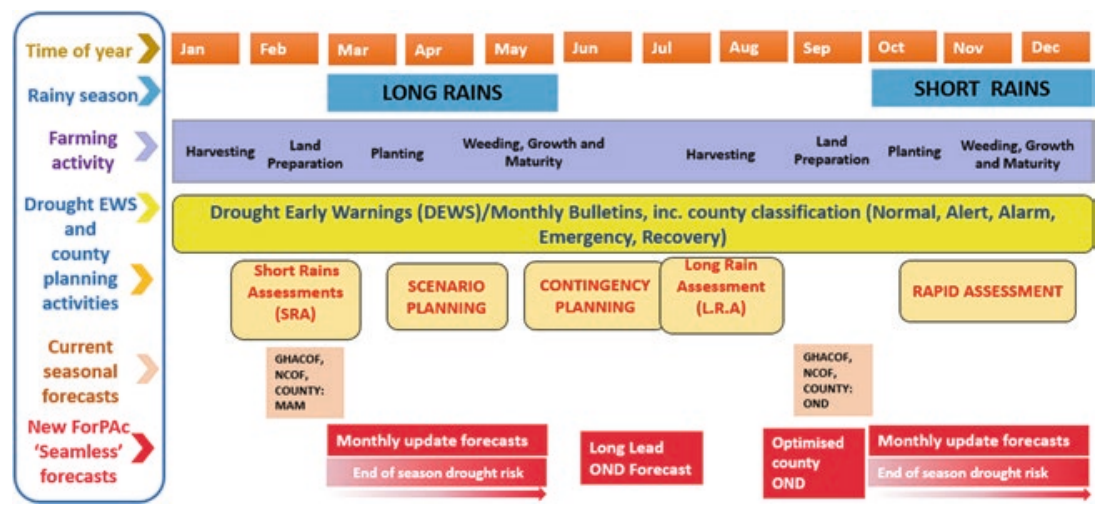

Fig. 4.1 Schematic showing seasonal: rains, farming activities, drought management activities, provision of climate information and key entry points where ForPAc seamless prototype forecast products could strengthen drought management and preparedness

PIPA analysis identified windows of opportunity for providing enhanced forecasts that could activate earlier drought mitigation and preparedness actions (see Fig. 4.1), and how climate information could better support each step within the DEWS process.

Working with the Kitui CSG, the project co-produced a suite of prototype products, including a long-lead seasonal forecast, an optimised seasonal and monthly forecast and a Standard Precipitation Index (SPI). These prototype forecast products were piloted for the 2019 October, November and December rains with decision-makers to consider what different preparedness actions could be applied recognising the probability and skill of the forecasts. Preparedness actions that aligned with the FREE principles, for forecasts, in this instance, indicating a $45-60 \%$ probability of above average seasonal rains, included: planting more maize than usual, vaccinating livestock against Rift Valley Fever, WASH sensitization, and desilting of water pans. ForPAc products were prototypes, and not yet official Kenya Meteorological Department products; because government ministries require official forecasts to justify action, some of the actions were taken and some remained proposed. While an effective framework for supporting elements of FREE, consideration of equity within PIPA could have been strengthened through participation from those people 
whose lives and livelihoods are directly affected by climate-related risks, thus enabling identification of innovative preparedness measures beyond those included within the County's existing Contingency Plan.

\section{Strengthening Flood-Resilient Urban Planning in Ouagadougou, Burkina Faso (AMMA-2050)}

Focused on enhancing understanding about High Impact Weather events to inform medium-term (5-50 years) decision-making in West Africa, the AMMA-2050 project has undertaken two pilot studies (in Burkina Faso and Senegal) to examine how tailored climate information can better support specific climate-sensitive decision-making processes. In Burkina Faso, partners have sought to strengthen flood-resilient urban planning for the capital, Ouagadougou, particularly within planning for the city's development, 'the Grand Ouaga plan,' in which participatory consultation has been limited.

PIPA was employed in both pilots to enable exploration of the views of different stakeholders, identification of additional partners who could support the aims of the project, and development of 'road maps' supporting a range of co-production processes led by different AMMA-2050 partners (Carter et al. 2019; see Chap. 3). In Burkina Faso, PIPA was used at different points of the project, to ensure different actors had spaces to discuss the FREE heuristics in relation to potential actions and outputs of the project. At the beginning of the project, in 2017, AMMA-2050 organised a joint workshop with the Building Resilience and Adaptation to Climate Extremes and Disasters (BRACED) Zaman Lebidi project, funded by the UK Department for International Development. The workshop focussed on how weather and climate information could support local government decision-making.

Prior to the PIPA problem tree process and visioning and stakeholder mapping exercises, the workshop promoted the equity principle through establishing a common ground amongst participating local government representatives, development actors, the national meteorological agency and partnering researchers. In this process, the local government decisionmaking context for mayors in rural and urban contexts was outlined, before providing an overview of key climate concepts and existing climate information services. Following this, participants engaged in a scenario exercise designed to strengthen decision-makers' confidence in using a 
range of climate products and support dialogue between decision-makers and technical experts. Simulating the difficulties of making appropriate use of climate information within commune-level decision-making processes, the exercise exemplified constraints in operationalising the heuristics of flexibility and robustness. In the final session of the workshop, AMMA-2050 and BRACED partners outlined how their projects could respectively strengthen effective use of climate information within local government decision-making. From this basis, AMMA-2050 then developed an Outcome Logic Model to guide the project's pilot in Ouagadougou. Most immediately, the PIPA Stakeholder mapping highlighted to AMMA-2050 partners the value of ensuring sustained engagement with local and national decision-makers. This resulted in the appointment of a dedicated focal point to ensure a channel for ongoing interaction with key stakeholders. While AMMA-2050 was focused on strengthening medium-term decision-making, stakeholders highlighted the need to also address more immediate climate-related risks. Consequently, partners developed an awareness raising pamphlet with advice on flood-preparedness and response, simultaneous with developing technical briefs on tools for supporting longer-term planning.

PIPA was also employed at the end of the project, in a very similar format. Participants coming from various branches of local and national government were asked to reflect on the impacts of AMMA-2050 on relevant policies and activities, focusing specifically on how project outputs could have contributed to reducing the usability gap in climate information. The stakeholder mapping highlighted complexities in hierarchies and scale that were acknowledged over the course of the project but never made explicit and discussed potential strategies for addressing those in a future, advocacy-focussed part of AMMA-2050. Flexibility and robustness of approaches was put forward as a key element of successful outputs; equity was mentioned especially in ensuring that approaches would not benefit a part of society while increasing risks for another. This workshop resulted in discussions among the societal partners focussing on the issue of bottom-up and top-down interventions, the potential of citizen-led action combined with project- or government-led ones, as well as the possibility of linking some more immediate flood awareness-raising initiatives to long-term national adaptation policies.

The participatory approaches employed by AMMA-2050 have offered spaces and illustrated ways of supporting more inclusive planning, 
including within the development of the Grand Ouaga plan, and the importance of recognising risks across decision-making levels and timeframes.

The use of PIPA in AMMA-2050 provided a shared learning experience for researchers and decision-makers to explore together how weather and climate information can better support local government decisionmaking. In doing so, it was an opportunity to practice the FREE heuristics and draw expertise for more integrated approaches to strengthening climate resilience. For participating early career climate scientists, the workshop provided a first experience to consider how their research could practically support decision-makers' concerns.

\section{Discussion of FREE as Framework to Support Climate-Resilient Decision-Making}

The underlying premise of using heuristics to support climate risk management is that the prediction and projection of the impacts of both shortterm high-impact weather events and longer-term climate changes are characterised by uncertainty. Within this context of uncertainty, the FREE framework provides guiding principles to help decision-makers derive mitigation, preparedness and adaptation actions that consider current knowledge of weather and climate change impacts. FREE provides a framework to support consideration of climate-related risks across timeframes, decision-making processes, sectors and social groups, while profiling the importance of equity considerations.

It is vital that decision-makers ensure flexibility to be able to take appropriate anticipatory and adaptation actions dependent on current and emerging scientific understanding of climate-related risks across timeframes. Given the inherent uncertainty of climate information, decisions need to be robust to the evolving 'envelope of uncertainty.' Actions taken in resource-constrained environments need to ensure economic no/low regrets and consider how measures to support mitigation and preparedness for immediate climate-related risks contribute to longer term climateresilient, sustainable development. The experience outlined earlier highlights the need to ensure that addressing climate-related risks is equitable, and that this is made explicit and includes inclusive decision-making in deciding the trade-offs across timeframes, sectors and social groups. 
PIPA has supported inclusive and transparent dialogue in planning for climate-related risks across urban and rural contexts and across timeframes. In both AMMA-2050 in Burkina Faso and ForPAc in Kenya, the PIPA methodology has enabled research to be better aligned with immediate and longer-term societal concerns and decision-makers' priorities. The use of PIPA in these research projects has demonstrated that the approach offers opportunities for supporting flexible, robust, low-regrets and equitable decision-making. It supported inclusive and participatory dialogue across researchers and decision-makers and helped to recognise trade-offs between short- and long-term objectives and between different elements of the FREE framework.

While providing a useful approach for considering the underpinning FREE principles, PIPA is shaped by pre-existing partnerships and networks, as much as it also offers opportunities for reshaping these. More widely the FREE framework provides a useful reference for assessing the extent to which approaches employed within climate-resilience strengthening initiatives have been able to operationalise its four guiding principles. As such, the FREE framework may be reviewed and further developed as a foundational tool for strengthening climate risk management.

\section{REFERENCES}

Agrawal, A., Kononen, M., \& Perrin, N. (2009). The role of local institutions in adaptation to climate change. Social Development Working Paper Series 118. Washington, DC: World Bank.

Briley, L., Brown, D., \& Kalafatis, S. E. (2015). Overcoming barriers during the co-production of climate information for decision-making. Climate Risk Management, 9, 41-49.

Carter, S., Steynor, A., Vincent, K., Visman, E., \& Waagsaether, K. (2019). Coproduction of African weather and climate services. Manual. Cape Town: Future Climate for Africa and Weather and Climate Information Services for Africa. Retrieved from https://futureclimateafrica.org/coproduction-manual Cash, D. W., Clark, W. C., Alcock, F., Dickson, N. M., Eckley, N., Guston, D. H., Jäger, J., \& Mitchell, R. B. (2003). Knowledge systems for sustainable development. Proceedings of the National Academy of Sciences, 100(14), 8086-8091.

Daly, M., \& Dilling, L. (2019). The politics of 'usable' knowledge: Examining the development of climate services in Tanzania. Climatic Change, 157, 61-80. https://doi.org/10.1007/s10584-019-02510-w. 
Daron, J. D., Sutherland, K., Jack, C., et al. (2015). The role of regional climate projections in managing complex socio-ecological systems. Regional Environmental Change, 15, 1-12. https://doi.org/10.1007/ s10113-014-0631-y.

Dayamba, D. S., Ky-Dembele, C., Bayala, J., Dorward, P., Clarkson, G., Sanogo, D., Mamadou, L. D., Traoré, I., Diakité, A., Nenkam, A., \& Binam, J. N. (2018). Assessment of the use of participatory integrated climate services for agriculture (PICSA) approach by farmers to manage climate risk in Mali and Senegal. Climate Services, 12, 27-35.

Dilling, L., \& Lemos, M. C. (2011). Creating usable science: Opportunities and constraints for climate knowledge use and their implications for science policy. Global Environmental Change, 21(2), 680-689.

Fox, G., \& Kniveton, D. (2018). People, participation, \& pathways. AMMA-2050 technical report 8.

Kirchhoff, C. J., Lemos, M. C., \& Kalafatis, S. (2015). Narrowing the gap between climate science and adaptation action: The role of boundary chains. Climate Risk Management, 9, 1-5.

Leitch, A. M., Palutikof, J. P., Rissik, D., Boulter, S. L., Tonmoy, F. N., Webb, S., Vidaurre, A. P., \& Campbell, M. C. (2019). Co-development of a climate change decision support framework through engagement with stakeholders. Climatic Change, 153(4), 587-605.

Lemos, M. C., Kirchhoff, C. J., \& Ramprasad, V. (2012). Narrowing the climate information usability gap. Nature Climate Change, 2(11), 789-794.

Lorenz, S., Dessai, S., Forster, P. M., \& Paavola, J. (2017). Adaptation planning and the use of climate change projections in local government in England and Germany. Regional Environmental Change, 17(2), 425-435.

Lorenzoni, I., Jordan, A., O’Riordan, T., Turner, R. K., \& Hulme, M. (2000). A co-evolutionary approach to climate change impact assessment-Part II: A scenario-based case study in East Anglia (UK). Global Environmental Change, $10(2), 145-155$.

Maier, H. R., Guillaume, J. H., van Delden, H., Riddell, G. A., Haasnoot, M., \& Kwakkel, J. H. (2016). An uncertain future, deep uncertainty, scenarios, robustness and adaptation: How do they fit together? Environmental Modelling o Software, 81, 154-164.

Niang, I., Ruppel, O. C., Abdrabo, M. A., Essel, A., Lennard, C., Padgham, J., \& Urquhart, P. (2014). Africa. In V. R. Barros et al. (Eds.), Climate change 2014: Impacts, adaptation, and vulnerability. Part B: Regional aspects. Contribution of working group II to the fifth assessment report of the intergovernmental panel on climate change. Cambridge University Press.

Pielke, R. A. Sr., Wilby, R., Niyogi, D., Hossain, F., Dairuku, K., Adegoke, J., Kallos, G., Seastedt, T., \& Suding, K. (2012). Dealing with complexity and 
extreme events using a bottom-up, resource-based vulnerability perspective. In A. S. Sharma, et al. (Eds.), Extreme events and natural hazards: The complexity perspective. Geophysical Monograph Series 196. https://doi.org/10.1029/ 2011 GM001086

Preston, B. L., Mustelin, J., \& Maloney, M. C. (2015). Climate adaptation heuristics and the science/policy divide. Mitigation and Adaptation Strategies for Global Change, 20(3), 467-497.

Ranger, N., Reeder, T., \& Lowe, J. (2013). Addressing 'deep' uncertainty over long-term climate in major infrastructure projects: Four innovations of the Thames Estuary 2100 project. EURO Journal on Decision Process, 1, 233-262. https://doi.org/10.1007/s40070-013-0014-5.

Roudier, P., Sultan, B., Quirion, P., \& Berg, A. (2011). The impact of future climate change on West African crop yields: What does the recent literature say? Global Environmental Change, 21(3), 1073-1083.

Shreve, C. (2016). Economic efficiency or gender equality: Conceptualizing an equitable "social framing" for economic evaluations to support gender equality in disaster risk- and environmental-management decision-making. Resources, 5, 25.

Singh, C., Daron, J., Bazaz, A., Ziervogel, G., Spear, D., Krishnaswamy, J., Zaroug, M., \& Kituyi, E. (2018). The utility of weather and climate information for adaptation decision-making: current uses and future prospects in Africa and India. Climate and Development, 10(5), 389-405. https://doi.org/ $10.1080 / 17565529.2017 .1318744$

Stainforth, D. A., Allen, M. R., Tredger, E. R., \& Smith, L. A. (2007). Confidence, uncertainty and decision-support relevance in climate predictions. Philosophical Transactions of the Royal Society A, 365, 2145-2161. https://doi.org/10.1098/ rsta.2007.2074.

Sultan, B., Defrance, D., \& Iizumi, T. (2019). Evidence of crop production losses in West Africa due to historical global warming in two crop models. Scientific Reports, 9, 12834. https://doi.org/10.1038/ s41598-019-49167-0.

Turnhout, E., Metze, T., Wyborn, C., Klenk, N., \& Louder, E. (2020). The politics of co-production: Participation, power, and transformation. Current Opinion in Environmental Sustainability, 42, 15-21.

United Nations International Strategy for Disaster Reduction (UNISDR). (2011). Briefing note 4: Effective measures to build resilience in Africa to adapt to climate change (Vol. 8). Geneva: UNISDR.

Vincent, K., Daly, M., Scannell, C., \& Leathes, B. (2018). What can climate services learn from theory and practice of co-production? Climate Services, $12,48-58$. 
Watkiss, P., Hunt, A., Blyth, W., \& Dyszynski, J. (2015). The use of new economic decision support tools for adaptation assessment: A review of methods and applications, towards guidance on applicability. Climatic Change, $132(3), 401-416$.

Wilby, R. L., \& Dessai, S. (2010). Robust adaptation to climate change. Weather, 65(7), 180-185.

Wilkinson, E., Weingartner, L., Choularton, R., Bailey, M., Todd, M., Kniveton, D., \& Cabot Venton, C. (2018). Forecasting hazards, averting disasters: Implementing forecast-based early action at scale. Technical Paper. London: Overseas Development Institute.

Open Access This chapter is licensed under the terms of the Creative Commons Attribution 4.0 International License (http://creativecommons.org/licenses/ by $/ 4.0 /$ ), which permits use, sharing, adaptation, distribution and reproduction in any medium or format, as long as you give appropriate credit to the original author(s) and the source, provide a link to the Creative Commons licence and indicate if changes were made.

The images or other third party material in this chapter are included in the chapter's Creative Commons licence, unless indicated otherwise in a credit line to the material. If material is not included in the chapter's Creative Commons licence and your intended use is not permitted by statutory regulation or exceeds the permitted use, you will need to obtain permission directly from the copyright holder.

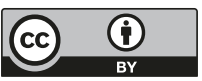

